\title{
Recent Electroweak Results from the Tevatron
}

\author{
Junjie Zhu ${ }^{1}$ \\ State University of New York at Stony Brook, Stony Brook, NY, 11794
}

\begin{abstract}
I present the recent electroweak measurements related to single $W, Z$ boson and diboson productions from the CDF and DØ experiments at the Fermilab Tevatron collider.
\end{abstract}

Keywords: Electroweak measurements, $W$ and $Z$ bosons, diboson processes

PACS: 12.15.-y, 13.38.Be, 14.70.Fm, 13.85.Qk, 14.60.Cd, 13.40.Em, 14.70.Hp, 13.85.Qk

\section{INTRODUCTION}

$W$ and $Z$ bosons are mainly produced via quark-antiquark annihilations at the Fermilab Tevatron collider. Precision measurements with these gauge bosons provide us with high precision tests of the Standard Model (SM) as well as indirect search for possible new physics beyond the SM.

\section{$W$ BOSON PROPERTIES}

Precision measurement of $W$ boson mass $\left(M_{W}\right)$. In the SM, $M_{W}$ can be calculated using the electromagnetic coupling constant $\alpha$, the Fermi constant $G_{F}$ and the weak mixing angle $\sin ^{2} \theta_{W}$. It also receives quantum radiative corrections that depend on the top quark mass and the SM Higgs boson mass. A precise measurement of $M_{W}$ thus can be used to make constaints on the Higgs mass. To make the equal contribution to the Higgs mass uncertainty, we need to have $\Delta M_{W} \approx 0.006 \times \Delta M_{\text {top }}$. With the current world average value of $\Delta M_{\text {top }}=1.3 \mathrm{GeV}$ and $\Delta M_{W}=0.025 \mathrm{GeV}, \Delta M_{W}$ is the limiting factor for the Higgs mass constraint.

At the Tevatron, $M_{W}$ is extracted from a template fit to the transverse mass $\left(M_{T}\right)$, lepton transverse momentum $\left(p_{T}^{\ell}\right)$ and missing transverse energy $\left.\mathbb{H}_{T}\right)$ distributions in $W \rightarrow \ell v(\ell=e, \mu)$ events. The template distributions are generated using a parameterized Monte Carlo (MC) simulation program. This program uses the state-of-the-art $W$ and $Z$ $\mathrm{MC}$ event generator and simulates the complex detector acceptance and response effects. The parameters used in the simulation are mainly determined using the $Z \rightarrow \ell \ell$ events. The uncertainties on these detector smearing parameters and theoretical calculations (including QCD and electroweak corrections and also the parton distribution functions (PDFs)) are propogated to the uncertainty on the measurement of $M_{W}$.

Using $1 \mathrm{fb}^{-1}$ of Run II data [1], the DØ collaboration measured $M_{W}=80.401 \pm$ 0.021 (stat) \pm 0.038 (syst) $=80.401 \pm 0.043 \mathrm{GeV}$, the most precise measurement from

${ }^{1}$ For the CDF and DØ Collaborations 

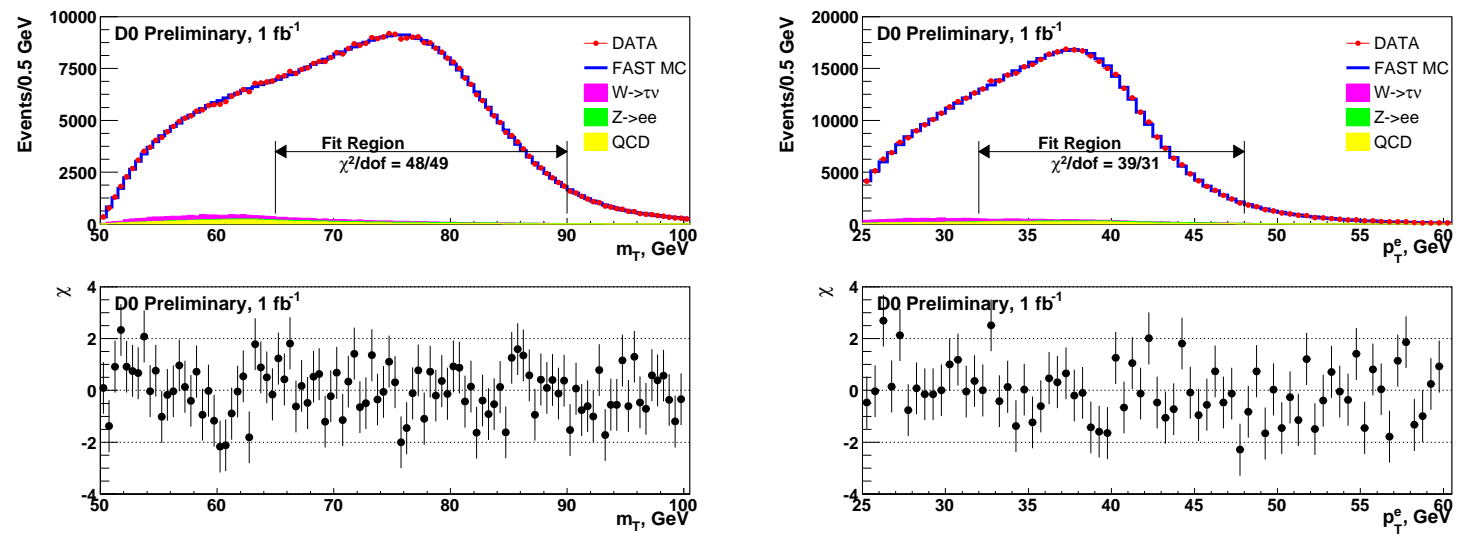

FIGURE 1. The $M_{T}$ and $p_{T}^{e}$ distributions for data and MC simulation with backgrounds added (top), and the difference between data and MC divided by the data uncertainty $(\chi)$ for each bin (bottom).

one single experiment to date. Figure 1 shows the data and $\mathrm{MC}$ comparison for $M_{T}$ and $p_{T}^{e}$ distributions.

Many of the systematic uncertainties in these measurements are limited by the size of the control samples (mainly $Z \rightarrow \ell \ell$ events) used to understand the detector and physics effects. These uncertaines can be improved with an analysis of the larger datasets in hand. CDF has begun analyzing a data sample with $\mathscr{L}=2.3 \mathrm{fb}^{-1}$ [2] and found the statistical uncertainty is 16 (15) $\mathrm{MeV}$ for muon (electron) channel and the uncertainty due to the lepton energy scale is 12 (20) $\mathrm{MeV}$ for muon (electron) channel. We expect to get the ultimate combined Tevatron measurement with an uncertainty of $15 \mathrm{MeV}$.

$W$ boson production charge asymmetry. At the Tevatron, $W^{+}\left(W^{-}\right)$bosons are produced primarily by the annihilation of $u(d)$ quarks in the proton with $\bar{d}(\bar{u})$ quarks in the antiproton. Due to the fact that $u$ quarks in the proton on average carry more momentum than $d$ quarks, $W^{+}\left(W^{-}\right)$boson tends to move along the proton (antiproton) direction, and thus results in an asymmetry in the $W$ boson rapidity distribution between $W^{+}$and $W^{-}$boson production. This asymmetry is sensitive to the PDFs which describes the fraction of momentum carried by each parton $(x)$ in the proton.

Since we can not measure the longitudinal momentum of the neutrino in $W \rightarrow \ell v$ decays, the $W$ charge asymmetry is traditionally measured as a function of the decay lepton pseudorapidity $(\eta)$. The $\mathrm{D} \varnothing$ collaboration analyzed $0.75 \mathrm{fb}^{-1}$ of data in electron channel and measured electron charge asymmetry for events with $E_{T}^{e}>25 \mathrm{GeV}, p_{T}^{v}>25$ $\mathrm{GeV}$ and electron $|\eta|<3.2$ [3]. The asymmetry is also measured in two electron $E_{T}$ bins to probe partons in different $x$ regions. The detector effects corrected charge asymmetry is compard to a NLO perturbative QCD calculation with CTEQ6.6 and MRST04 PDF sets. The measured charge asymmetries tend to be lower than the theoretical predictions for high rapidity electrons.

In a recent analysis using $1 \mathrm{fb}^{-1}$ of data by the CDF collaboration [4], the $W$ boson charge asymmetry is measured directly for the first time. The longitudinal momentum of the neutrino is estimated on an event-by-event basis using the $W$ boson mass constraint. The measured asymmetry is found to have good agreement with the predictions of a 
NNLO QCD calculation using the MRST 2006 NNLO PDF sets and a NLO QCD calculation using the CTEQ6.1 NLO PDF sets.

For both measurements, except the largest rapidity bin $(2.8<|\eta|<3.2)$ in the $\mathrm{D} \varnothing$ measurement, the overall experimental uncertainties are smaller than the uncertainties due to PDFs. These measurements can be used to make tighter constraints on the global PDFs fits and reduce the uncertainty on the future $W$ boson mass measurements.

\section{DIBOSON PRODUCTION}

The diboson production at the Tevatron is sensitive to the couplings between gauge bosons. These triple gauge couplings (TGCs) are a direct consequence of the nonAbelian group structure of the SM. Understanding diboson production is also critical for Higgs searches where dibosons are a major source of backgrounds in several important channels. The diboson measurements at the Tevatron often involve measurements of the overall production cross section and TGCs. The diboson processes with one charged $W$ boson in the final state are discussed here. The general Lorentz invariant effective Lagrangian describing $W W V(V=\gamma$ or $Z$ ) vertices has five independent parameters with the assumption of EM gauge invariance and $\mathrm{C}$ and $\mathrm{P}$ conservation. The five parameters are $g_{1}^{Z}, \kappa_{Z}, \kappa_{\gamma}, \lambda_{Z}$ and $\lambda_{\gamma}$. In the SM, $g_{1}^{Z}=\kappa_{Z, \gamma}=1$ and $\lambda_{Z, \gamma}=0$. These couplings are often written in terms of their deviations from their SM values as $\Delta g_{1}^{Z}=g_{1}^{Z}-1$ and $\Delta \kappa_{Z(\gamma)}=\kappa_{Z(\gamma)}-1$. A dipole form factor $\alpha(\hat{s})=\alpha_{0} /\left(1+\hat{s} / \Lambda^{2}\right)^{2}$ for one arbitrary coupling $\alpha$ is introduced to avoid the unitarity violation, where $\Lambda$ is the new physics energy scale, and the limits are set in terms of $\alpha_{0}$.

$W W \rightarrow \ell v \ell v . \quad$ Both CDF and DØ Collaborations select $W W$ events with two high

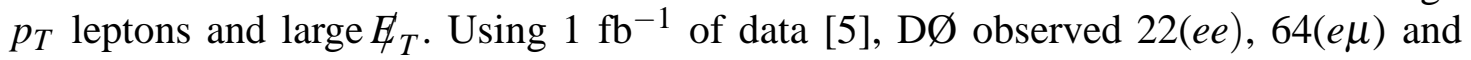
$14(\mu \mu)$ candidates. These numbers are consistent with the SM predictions of $23.5 \pm$ $1.9(e e), 68.6 \pm 3.9(e \mu)$ and $10.8 \pm 0.6(\mu \mu)$ events. The measured cross section is $11.5 \pm$ 2.1 (stat+syst) \pm 0.7 (lumi) pb, which is consistent with the SM prediction of $12.4 \pm 0.8$ pb. A similar measurement by CDF used $3.6 \mathrm{fb}^{-1}$ of data [6]. This measurement makes use of matrix element (ME) based likelihood ratios. Event kinematics are used to assign an event-by-event probability $\mathbf{P}$ based on leading-order ME cross section calculation for the $W W$ process and for the major background processes such as $W W, W \gamma$ and $W+$ jet. The background fractions are estimated using the GEANT MC simualtion, while the signal fraction is extracted using a likelihood fit to the $L R_{W W}$ distribution. $L R_{W W}$ is defined as $P_{W W} /\left(P_{W W}+\sum_{i} k_{i} P_{i}\right)$ with $k_{i}$ as the relative fraction of each background source, $P_{W W}$ and $P_{i}$ represent the event probability for $W W$ and each background process respectively. The measured cross section is $12.1 \pm 0.9(\mathrm{stat})_{-1.4}^{+1.6}(\mathrm{syst}) \mathrm{pb}$, which is again consistent with the SM prediction. DØ also used the leading and trailing lepton $p_{T} \mathrm{~S}$ to set limits on aTGCs assuming $\Lambda=2 \mathrm{TeV}$. The results are $-0.14<\Delta g_{1}^{Z}<0.30$, $-0.54<\Delta \kappa_{\gamma}<0.83$ and $0.14<\lambda_{Z}=\lambda_{\gamma}<0.19$ under the $S U(2)_{L} \times U(1)_{Y}$-conserving constraints, and $-0.12<\Delta \kappa_{Z}=\Delta \kappa_{\gamma}<0.35$, with the same $\lambda_{Z(\gamma)}$ limits as above, under the $W W \gamma=W W Z$ constraints. 
$W W / W Z \rightarrow \ell v j j . \quad$ The $\mathrm{D} \emptyset$ Collaboration recently reported the first evidence of $W W / W Z \rightarrow \ell v j j$ process at the hadron collider [7]. The signatures are one high $p_{T}$ lepton, large $Z_{T}$ and two high $p_{T}$ jets. The background arises mainly due to $W / Z+$ jets, QCD multijet, and $t \bar{t}$ events. A multivariate classifier (Random Forest) is used to separate the signal from backgrounds. The signal and background contents are determined by fitting the signal and background random forest templates to the data. The measured cross section is $20.2 \pm 2.5$ (stat) \pm 3.6 (syst) \pm 1.2 (lumi) pb, consistent with the SM prediction of $16.1 \pm 0.9 \mathrm{pb}$. The dijet $p_{T}$ spectrum is used to set aTGCs limits assuming $\Lambda=2 \mathrm{TeV}:-0.12<\Delta g_{1}^{Z}<0.19,-0.44<\Delta \kappa_{\gamma}<0.55$ and $-0.10<\lambda_{Z, \gamma}<0.11$ under the $S U(2)_{L} \times U(1)_{Y}$-conserving constraints, and $-0.16<\Delta \kappa_{Z, \gamma}<0.23$ and $-0.11<$ $\lambda_{Z, \gamma}<0.11$, under the $W W \gamma=W W Z$ constraints.

$W Z \rightarrow j j \ell \ell$. The CDF Collaboration also reported a search for anomalous $W Z$ production at the Tevatron using the two charged leptons, two jets final state [8]. Three bins in dilepton $p_{T}$ are used: $105-140 \mathrm{GeV}$ (control region), $140-210 \mathrm{GeV}$ (medium region) and $>210 \mathrm{GeV}$ (high region). Events in the control region are mainly used to validate data modeling and determine backgrounds. The dijet mass $M_{j j}$ for the medium and high regions are used to set limits on the cross section and aTGCs. The 95\% CL upper limit on the cross section is found to be $234 \mathrm{fb}$ and $135 \mathrm{fb}$ using events in the medium and high region respectively. Under the $W W \gamma=W W Z$ constraints, the limits

on the aTGCs are $-0.20<\Delta g_{1}^{Z}<0.29,-1.01<\Delta \kappa_{Z, \gamma}<1.27$ and $-0.16<\lambda_{Z, \gamma}<0.17$ assuming $\Lambda=2 \mathrm{TeV}$, and $-0.22<\Delta g_{1}^{Z}<0.32,-1.09<\Delta \kappa_{Z, \gamma}<1.40$ and $-0.18<$ $\lambda_{Z, \gamma}<0.18$ assuming $\Lambda=1.5 \mathrm{TeV}$.

\section{CONCLUSIONS}

With the increasingly large datasets, both $\mathrm{CDF}$ and $\mathrm{D} \varnothing$ continue to improve our understanding of electroweak production at the hadron collider.

\section{ACKNOWLEDGMENTS}

I would like to thank all my $\mathrm{CDF}$ and $\mathrm{D} \varnothing$ colleagues for their hard works, as well as the conference organizers for hosting an excellent conference.

\section{REFERENCES}

1. V.M. Abazov et al. (D0 Collaboration), http://www-d0.fnal.gov/Run2Physics/WWW/results/prelim/EW/E27.

2. T. Aaltonen et al. (CDF Collaboration), http://www-cdf.fnal.gov/physics/ewk/2008/wmass/

3. V.M. Abazov et al. (D0 Collaboration), Phys. Rev. Lett. 101, 211801 (2008).

4. T. Aaltonen et al. (CDF Collaboration), Phys. Rev. Lett. 102, 181801 (2009); A. Bodek et al., Phys. Rev. D 77, 111301(R) (2008).

5. V.M. Abazov et al. (D0 Collaboration), submitted to Phys. Rev. Lett., arXiv:0904.0673.

6. T. Aaltonen et al. (CDF Collaboration), http://www-cdf.fnal.gov/physics/ewk/wwllll/index.html

7. V.M. Abazov et al. (D0 Collaboration), Phys. Rev. Lett. 102, 161801 (2009).

8. T. Aaltonen et al. (CDF Collaboration), http://www-cdf.fnal.gov/physics/ewk/2008/WWZaTGC/ 